\title{
Analysis of the Backflow Recirculation in Flow Blurring Nozzles via Lagrangian Coherent Structures
}

\author{
Cihan Ates*, Valerio Giovannoni, Niklas Bürkle, Marc Keller, Max Okraschevski, Rainer Koch, \\ Hans-Joerg Bauer \\ Institute of Thermal Turbomachinery, Karlsruhe Institute of Technology, Karlsruhe, Germany \\ *Corresponding author email: cihan.ates@kit.edu
}

\begin{abstract}
The liquid atomization process relies on the disturbance of the liquid surface by various forces. In the case of "flow-blurring" (FB) atomization, this is achieved by inducing turbulence near the liquid channel exit. In this study, we analyze the underlying dynamics of these coherent turbulent structures and their role in the primary atomization within the FB regime. For that purpose, Smoothed Particles Hydrodynamics (SPH) simulations have been conducted using the geometry of an FB atomizer, which was also studied experimentally. An in-house developed visualization and data exploration platform (postAtom) was used to capture the time-resolved, Lagrangian coherent structures (LCSs) via the finite-time Lyapunov exponent (FTLE) fields. The results indicate that the design of the mixing chamber can trigger an oscillatory behavior at the nozzle exit, which has a direct impact on the evolution of the micro-ligaments and the consecutive primary atomization. It is further shown how the FTLE fields can be used as a guide to optimize the nozzle geometry.
\end{abstract}

\section{Keywords}

Primary breakup, flow blurring nozzle, shadowgraph, smoothed particle hydrodynamics, Lagrangian coherent structures.

\section{Introduction}

Atomization efficiency of liquids plays an important role in several processes, in particular combustion where the size of droplets directly affects the chemical reaction and the formation of pollutants. Most injectors for gas turbines nowadays are based on the "air-blast" (AB) principle, where a liquid jet is broken up by shear forces due to the interaction between liquid and a high velocity gas. Alternatives are internal mixing nozzles, such as "effervescent nozzles", where the low velocity gas interacts with the liquid bulk internally, upstream of the nozzle discharge orifice. Despite their ability to produce fine droplets, the effervescent atomizers are not as widespread as air-blast nozzles due to the need for air being compressed to the same pressure level as the liquid [1], which requires a considerable amount of energy. This is especially true in the case of gas turbines.

Recent interest has been focusing on a new type of air assisted nozzle, called "flow-blurring" (FB) atomizer, which features a working principle common to both air-blast injectors and effervescent injectors. In this configuration the liquid flows through a cylindrical tube with an internal diameter (d) and the atomizing gas is forced through the gap with height (h) between the exit section of the nozzle and the tip of the cylindrical liquid tube. It was observed that when the ratio $\mathrm{h} / \mathrm{d}$ was higher than 0.25 , the spray had a similar shape and characteristic of a typical air-blast atomizer [2]. On the contrary, when the value of this ratio dropped below 0.25 , a recirculation of the atomizing air into the liquid bulk was observed, along with a different shape of the spray and the production of finer droplets. The literature available to date $[3,4,5,6]$ has demonstrated the performance of FB injection being comparable or even better to those of an air-blast nozzle.

Since its discovery, it has been known that the transition from the conventional flow focusing 
pattern to the flow blurring regime is triggered at a certain geometric configuration and the gas-to-liquid ratio (GLR), which forces the gas phase to flow partially back into the liquid channel, creating the back-flow mixing region. This internal flow recirculation is characterized by the gas penetration depth, GLR and the geometry itself. Nevertheless, the dynamic nature of the turbulent structures, which in fact generate the transient micro-scale ligaments, hence the blurred plume, has not been investigated up to now. In this study, we explore the evolution of the backflow recirculation zone and its dependency on the nozzle geometry in order to clarify the underlying momentum transfer mechanism and its influence on the primary atomization.

\section{Methods}

\section{Smoothed Particles Hydrodynamics (SPH)}

The atomization process is simulated from the first principles using SPH method due to its inherent advantages over grid-based methods, particularly for the prediction of primary atomization. The main idea behind SPH is to evaluate the physical property of a particle or its spatial derivative by interpolating over the neighboring particles within a certain radius of influence. Because of its fully Lagrangian nature, the SPH method can naturally track the gas-liquid interfaces by the movements of uniquely defined particles (liquid/gas), even in the presence of large deformations as particles move according to the actual fluid velocity.

In the present study, the in-house developed code turboSPH is adopted to model the two phase flow within the FB nozzles. In detail, the continuity equation is solved as introduced by Espanol et al. [7] to be able to handle large mass discontinuities (see [8] for details). The symmetric scheme as proposed by Monaghan [9] is used to discretize the pressure gradient as it conserves linear momentum. Following the weakly compressible approach, the pressure is determined by means of Tait equation with additional background pressure to avoid tensile instability [10]. Surface tension is represented by the Continuum Surface Force model [11]. It should be noted that the suitability of this modeling strategy has already been demonstrated for the primary breakup of liquids in realistic spray nozzle configurations at both atmospheric and high pressure conditions. [12, 13, 14, 15].

\section{LCS and FTLE}

In the pioneering work of Haller [16], he proposed to identify Lagrangian-Coherent Structures (LCS) and to interpret them as a coherent vortex structure. In the same study, he further demonstrated the ridges in the fields of the so called Finite-Time Lyapunov Exponent (FTLE) enable the identification of LCS [16]. FTLE is based on a flow map $\phi_{t_{0}}^{t}\left(x_{0}\right)$ that associates a position $x_{0}$ at time $t_{0}$ with a position $x$ at time $t$. Accordingly, the gradient of $\phi_{t_{0}}^{t}\left(x_{0}\right)$ gives an indication on the deformation of the continuum in the vicinity of $x_{0}$ between $t_{0}$ and $t$. Herein, the eigenvalues $(\lambda)$ of $\nabla \phi_{t_{0}}^{t}\left(x_{0}\right)$ provide an objective measure of the local expansion or contraction, where the FTLE is defined as scaled and normalized value of the largest eigenvalue $\lambda_{n}\left(x_{0}, t_{0}, t\right)$ :

$$
F T L E_{t_{0}}^{t}\left(x_{0}\right)=\frac{1}{\left|t-t_{0}\right|} \log \left(\sqrt{\lambda_{n}\left(x_{0}, t_{0}, t\right)}\right) .
$$

The time difference $\left|t-t_{0}\right|$ is the reference time span $(\Delta t)$ and needs to be adjusted individually according to the time scale of the underlying flow phenomenon [13]. If $t>t_{0}$ the FTLE is called "forward in time" FTLE $^{+}$and for $t<t_{0}$ "backward in time" FTLE ${ }^{-}$respectively. The $\mathrm{FTLE}^{+}$measures the rate of separation, while the $\mathrm{FTLE}^{-}$measures the attraction between two infinitesimally close particles advected over $\Delta t$. In this study, $\mathrm{FTLE}^{-}$are utilized to track the coherent structures around the FB nozzles with a $\Delta t$ of $100 \mu \mathrm{s}$. In order to efficiently compute FTLE fields based on SPH data sets, the interactive data exploration environment "postAtom" mainly developed and implemented by the Institut für Visualisierung und Datenanalyse (IVD) in joint cooperation with ITS [13] was employed. 


\section{Computational Setup}

The base geometry of the nozzle considered in this work is the $2 \mathrm{D}$ representation of the modular FB nozzle designed for the experimental investigations conducted in parallel (Fig. 1). It consists of one central cylindrical and one concentric annular flow duct, referred as the liquid channel and the annular air duct, respectively. The nozzle is designed in a way that the gap between the liquid channel and nozzle exit $(\mathrm{h} / \mathrm{d})$ can be adjusted at will.
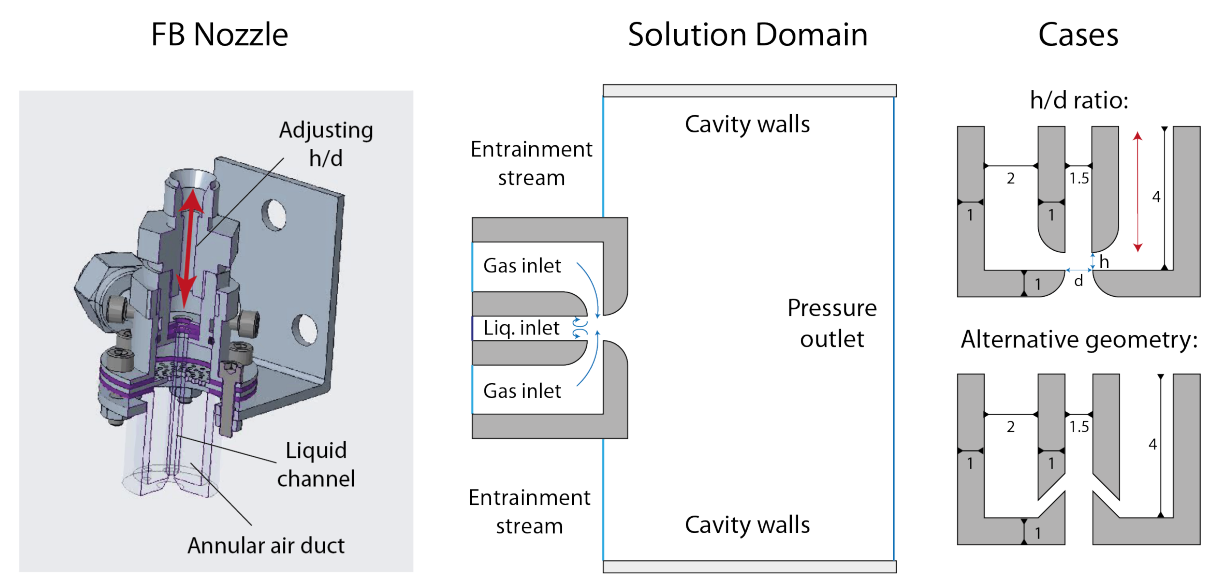

Figure 1. Nozzle Geometry and the corresponding computational domain of the base geometry

The domain for the SPH predictions is also shown in Fig. 1. It consists of the FB injector placed in a cavity extending $8.0 \mathrm{~mm}$ downstream of the nozzle with a width of $19.5 \mathrm{~mm}$. Dimensions of the simulated cavity depth, the heights of the annular flow duct and the nozzle were optimized with a preliminary study to ensure (i) atomizing air and liquid inlets are not disturbed by the radial flow at the exit and (ii) the advection of LCS without the influence of exit pressure boundary condition. In other words, the size of the computational domain was gradually reduced by monitoring the gas flow before conducting extended simulations for FTLE calculations.

Atomizing air ( 4 bar) enters the computational domain through two inlet boundaries located above and below the liquid channel walls. The piecewise linear velocity profiles are used to mimic the boundary layers closed to the walls. The boundary layer thickness is based on previous velocity profile measurements for similar flow conditions [14]. Following the boundary layers, free stream velocities are imposed in the flow direction considering turbulent flow conditions. The liquid film enters the channel with a laminar velocity profile. The mean velocities of the atomizing air is calculated based on a GLR of 10, nozzle geometry and liquid mass flow rate of $10 \mathrm{~g} \mathrm{~min}^{-1}$. An entrainment velocity of $16 \mathrm{~m} / \mathrm{s}$ is imposed on the sides of the nozzle (i) to mimic the potential closed chamber applications, (ii) to reduce the influence of the artificial recirculation zones in the cavity and and to ensure a steady outflow velocity at the outlet. Noslip boundaries are imposed at the walls of the injector while cavity walls are represented by the slip boundary conditions.

Three groups of simulations were performed for: (i) the extended analysis of flow-blurring conditions on the base geometry, (ii) the role of $\mathrm{h} / \mathrm{d}$ ratio on the back-mixing phenomena and (iii) the impact of geometry on mixing patterns by using an alternative geometry (Fig. 1). The space was discretized by using a mean inter-particle distance of $5 \mu \mathrm{m}$, leading to 4 million particles for each case. Both the cavity and the nozzle were initially filled with gas particles. All the simulations were run in parallel on 240 processors. The base geometry was simulated for 138,240 CPU-hours in order to analyze the converged statistics for the atomization process, yielding a physical time of $77 \mathrm{~ms}$. Ensuring the convergence rate, the rest of the simulations were run for 86,400 CPU-hours on 240 processors. The mean time step $d_{t}$ was $5 \mathrm{~ns}$ in all cases. The solutions were stored at a rate of $200 \mathrm{kHz}$ to meet the high spatial and temporal resolution 
requirements of the FTLE calculations.

For the sake of a more comprehensible visualization of vortex structures and the opportunity to provide a much finer spatial resolution of the flow field, the current study is based on SPH computations in 2D. Due to this simplification, fluctuations and instabilities in circumferential direction can not be captured. However, the general conclusions drawn from this study can analogously be transferred to 3D, which will be the focus of a follow-up study (Please refer to Dauch et al. [17] for a comparison between 2D and 3D simulations of primary breakup in airblast atomizers).

\section{Results and discussion \\ Backflow recirculation in FB Regime}

In an attempt to analyze the development of the FB regime, we first look at the base geometry with $G L R=10$ and $h / d=0.20$, which falls into the flow-blurring regime. Development of the gas-liquid (G-L) interface and the interaction between the nozzle geometry are illustrated with the FTLE- fields in Figure 2, for which the corresponding gas-liquid interfaces are indicated with dashed curves.

Herein the early time solutions $(t<0.03 \mathrm{~s}$ ) provide valuable insights into the interaction between nozzle geometry and atomizing air. It was seen that (Fig. 2 (a-b)) the internal mixing of the gas phase at the nozzle exit leads to oscillatory vortex shedding events, even in the absence of the liquid phase. In time, gaseous bubbles evolved in the liquid channel, where the coherent structures cycle between large ' $O$ ' shape vortices and symmetric ' 8 ' shape vortex rollers (Fig.2(c-e)). At a closer look, we observe that radially inward gas flow converges into a coherent "highway", along which the atomizing air flows preferably. These counter-current flowing gas streams (i) mix around the orifice exit, (ii) create liquid channel vortices, (iii) interact with the wakes formed by the separated flow around the corners and create nonlinear perturbations upstream of the exit point (Fig.2(a-e)). These interactions trigger a greater cyclic event that leads to the oscillatory vortex shedding observed at larger time scales. In particular, the origin of the vortex shedding events can be traced back to the mixing chamber, where the vortices are "nucleated" by the radially inward gas flow. In the following steps, these nucleation points are typically advected by the bulk flow towards the orifice exit walls (Fig.2(c-e)). Accordingly, the shape of the exit orifice has an indirect impact on the oscillatory behavior of the vortex shedding, as it influences how these coherent structures interact with each another and with the nozzle walls. As the G-L interface gets closer to the mixing zone, a transient period was identified, where large bubbles are formed and collapsed (Fig.2(d-f)). These events disappear after reaching the steady conditions, after which a competitive, cyclic behavior involving both phases dominates the atomization process throughout the simulation.

These cyclic events are triggered when the G-L interface is relatively flattened (by the previous events) at the same height with the gaseous flow inlet (Fig. 2(g)). The direct interaction between the radial gas flow and the liquid surface leads to the formation of micro-ligaments at the liquid surface, closer to the gas inlet points in the mixing zone (Fig. 2(g)). These small structures grow as they are pushed towards to the center-line by the gaseous phase (Fig. 2(h,i)). This wave propagation is similar to what we observed during the crescent formation under shearing gas flow in air-blast atomizers [13]. This interaction also affects (i) the evolution of "coherent gas flow highways" and (ii) how they interact with one another and the orifice walls. In particular, the symmetry history of the coherent structures creates a bifurcation: if the history of the competing LCSs are relatively symmetric, these waves merge into a large, 'W' shaped ligament around the center-line (Fig. 2(j)). If the symmetry is preserved in the next time steps, liquid mass flow flattens the 'W' shape into a relatively flat '-' interface profile, completing the small cycle (Fig. 2(g)). If the distribution of the vortex rollers are not symmetric during the wave collapse, which was the more common case in the simulations, the asymmetry pushes the wave towards the weaker end, closing the gas exit (Fig. 2(k)). This further triggers a second 

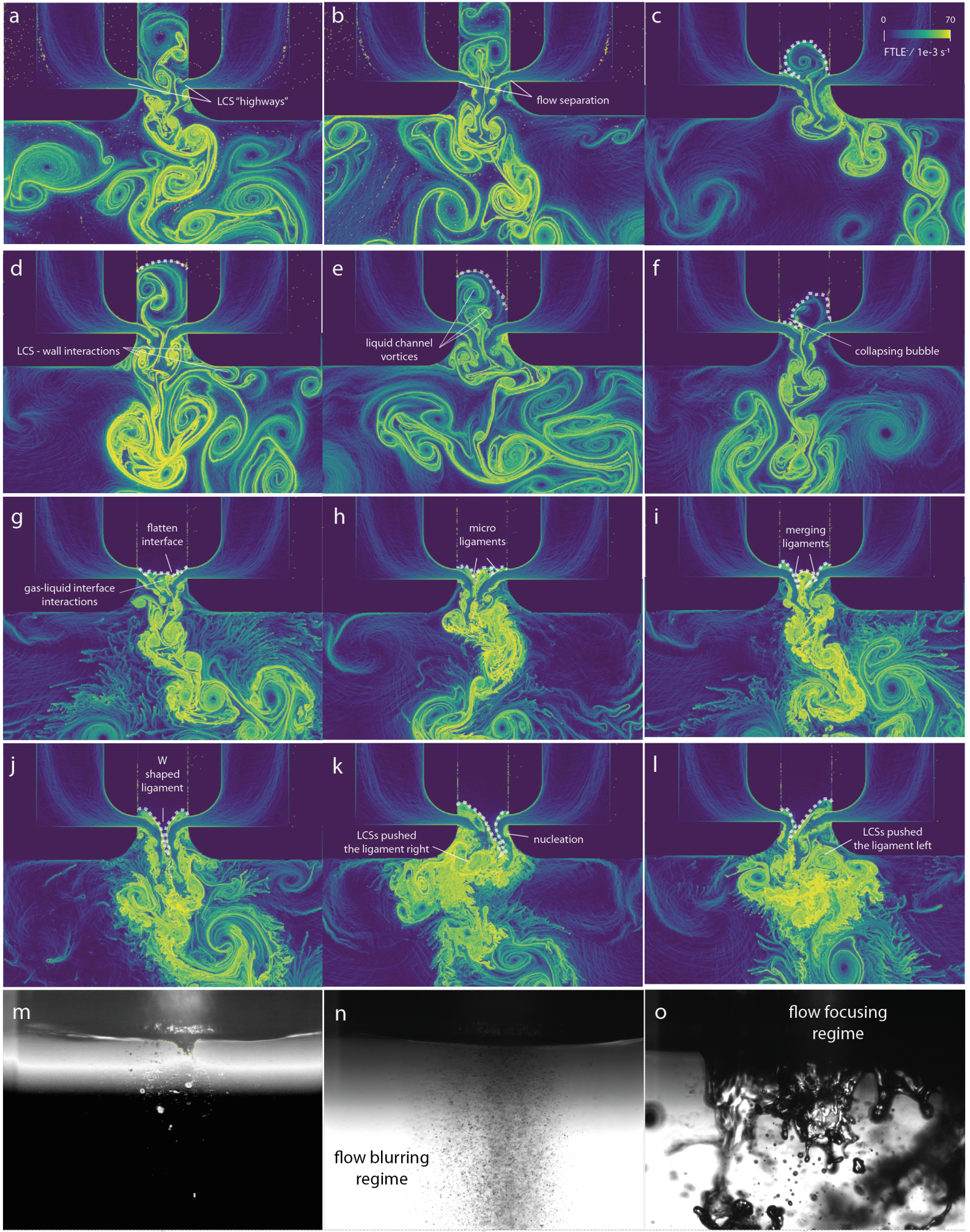

Figure 2. Instantaneous FTLE- fields( FTLE-: Backward in time Finite-Time Lyapunov Exponent) around the base geometry with shadowgraph images. Evolution of the coherent structures can be traced from the corresponding video archive. 
series of events, during which the gas flow rolls over itself between the liquid ligament and the orifice wall (Fig. $2(\mathrm{k}, \mathrm{l})$ ). As the coherent structures grow stronger, they push the large ligament to the preceding stronger end (Fig. 2(I)). During this "endless battle", ligaments exhibit an oscillatory behavior, occasionally equilibrate the strengths of the vortices and reform the centered 'W' shaped ligament. Here the meta-stable state is either broken by gas flow - wall interactions, or the continuous liquid flow flattens the 'W' and closes the larger cycle. Statistically, these competing forces are found to be the primary cause of the macroscopic oscillatory behaviour where the largest ligaments are carried around the nozzle exit, drifting between - $R$ to $+R$, around the centerline of the orifice. This virtual observation is also confirmed by the shadowgraph experiments. The tip of the 'W' shape ligament was observed occasionally at the exit of the liquid channel (Fig. 2(m)), which typically moved around the centerline. Immediately downstream of the nozzle, the FB atomization regime was achieved experimentally with the base case flow settings (Fig. 2(n)). As a comparison, the flow focusing regime with the same geometry and optical settings is shown in Fig. 2(0). When the flow regime is shifted by lowering the GLR and/or increasing the $\mathrm{h} / \mathrm{d}$ ratio, the ligaments grew drastically in size and the FB plume completely disappeared.
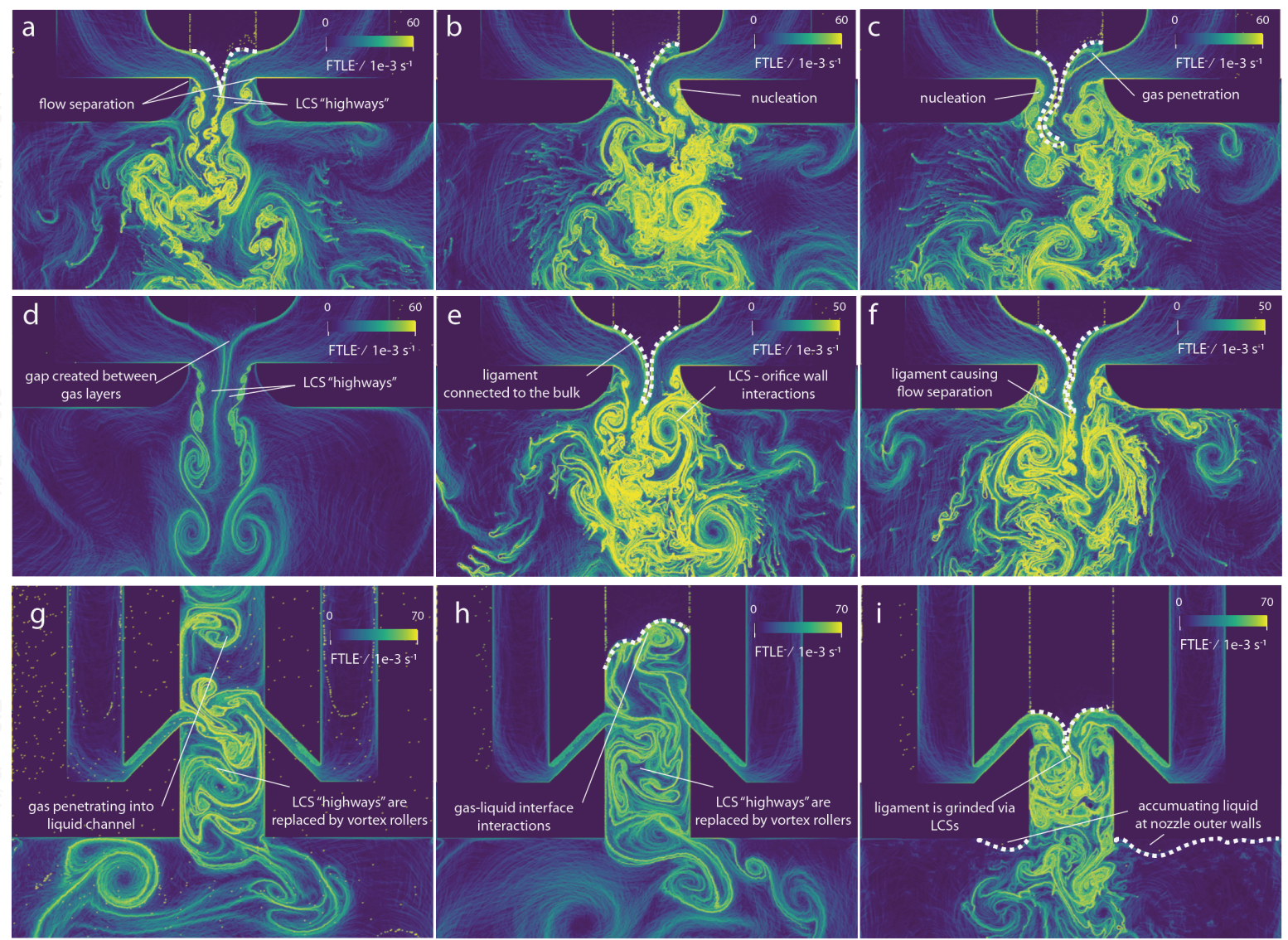

Figure 3. Influence of the $\mathrm{h} / \mathrm{d}$ ratio and the nozzle geometry on back-mixing phenomena. Evolution of the coherent structures can be traced from the corresponding video archive.

\section{Influence of $h / d$ ratio on the back-mixing phenomena}

Another interesting finding is the role of $h / d$ ratio on the interactions between the G-L interface, radial gas flow and the mixing chamber geometry. Herein, we present two additional cases with $\mathrm{h} / \mathrm{d}$ ratios of 0.4 and 0.6 , referred as Case II and Case III in the following discussions.

When $\mathrm{h} / \mathrm{d}$ ratio is increased from 0.2 to 0.4 , the formation of liquid channel vortices are rarely observed at the early transient stage $(t<0.03 \mathrm{~s})$ and they barely penetrate into the liquid channel. 
After reaching "steady" conditions, one large ligament is formed in the shape of ' $\Lambda$ ', unlike the 'W' shaped ligaments seen in FB regime (Fig. 3(a)). In other words, surface stripping and gas penetration into the liquid channel are very limited. It is also seen that the LCS highways of the gas flow mix further away from the nozzle (Fig. 3), yet still interacts with the nozzle outer walls and the orifice exit, which in turn affects the detachment and spread of the large ligament (Fig. 3(b,c)). The ' $\Lambda$ ' shaped ligament is noticeably larger and stretched outside the orifice exit periodically. When the h/d ratio is further increased (Case III), liquid channel vortices are not observed at all, as the gas mixing point is further advected towards nozzle downstream. The liquid phase does not interact with gas "LCS highways" and rather fills the gap left between these dynamical boundaries (Fig. 3(d)). Moreover, the LCSs act like a prefilmer for the growing ligament that stretches towards the nozzle exit, for which the disintegration process is mainly governed by the outer wall-vortex interactions Fig. 3(e,f)). It should also be noted that the ligament is found to be the thickest and the largest for Case III, root of which is always attached to the bulk liquid phase (Fig. $3(e, f)$ ). In this regard, the atomization process looks very similar to what is observed in a prefilming air-blast atomizer.

\section{Impact of geometry on mixing patterns}

In an attempt to explore better mixing strategies and to illustrate the impact of nozzle geometry on the back-mixing phenomena, we tested an alternative geometry with a $\mathrm{GLR}=10$ and $\mathrm{h} / \mathrm{d}=0.20$. By directing the gas flow 45 degree inwards (Fig. 1), the gas phase can be forced towards the liquid channel as expected (Fig. 3(g)), which continuously interacts with the G-L interface during the transient period (Fig. 3(h)). At "steady" state, a single ligament is attached to the bulk liquid phase at the gas inlet height, similar to the previous cases. In this modified geometry, however, the ligament size is comparably smaller. More importantly, the gap between the G-L interface and the nozzle exit is consistently filled with LCSs rolling over one another, which grinds the ligament into fine droplets within the nozzle (Fig. 3(i)). Nonetheless, these fine droplets are carried towards to the orifice walls with the same LCSs, and consequently being accumulated at the outer walls of the nozzle (Fig. 3(i)). In due time, the accumulated liquid is expected to detach as large ligaments, which is obviously not undesirable. The preliminary tests presented here indicate that there is a strong relationship between the (i) back-mixing phenomena and (ii) the gas mixing strategy, orifice geometry, h/d ratio and the wall-LCS interactions both inside and outside of the nozzle. The turbulence driven atomization process in the FB nozzles seems to be much more complex than the hypothesized mixing patterns in previous studies, hence a structured shape optimization study is needed to improve the performance of the generic FB nozzle geometry.

\section{Conclusions and Outlook}

In the present work, our objective is the qualitative analysis of the backflow recirculation phenomena in flow blurring nozzles. For that purpose, SPH simulations with very high spatial and temporal resolution were conducted to extract FTLE fields, which were used to explore the evolution of the LCSs inside the nozzle. Our analysis showed that the $\mathrm{h} / \mathrm{d}$ ratio determines (i) how the "coherent gas flow highways" are created, (ii) the stability of the micro ligaments formed on the gas-solid interface, (iii) how these small structures coalesce into a single, large ligament, (iv) interaction between the orifice geometry and the gas-liquid interface, ( $v$ ) the size of the ligaments stretching out of the nozzle, (vi) the frequency of vortex shedding and the consecutive primary atomization. We also tested an alternative geometry with the objective to achieve better gas mixing and demonstrated that the gas mixing strategy must be coupled with the annular gas chamber design to alleviate the liquid accumulation in the dead zones, which act like secondary sources of large, detached ligaments. The future work includes the quantitative analysis of the two phase flow and the FTLE fields in the primary atomization zone, where the outcomes will be used to optimize the chamber geometry. 


\section{References}

[1] Sovani, S., Sojka, P., and Lefebvre, A., 2001. "Effervescent atomization". Progress in Energy and Combustion Science, 27(4), pp. 483-521.

[2] Gañán-Calvo, A. M., 2005. "Enhanced liquid atomization: From flow-focusing to flowblurring". Applied Physics Letters, 86(21), may, pp. 1-3.

[3] Simmons, B. M., Panchasara, H. V., and Agrawal, A. K., 2009. "A comparison of air-blast and flow-blurring injectors using phase Doppler particle analyzer technique". In Proceedings of the ASME Turbo Expo, Vol. 2, American Society of Mechanical Engineers Digital Collection, pp. 981-992.

[4] Jiang, L., and Agrawal, A. K., 2015. "Spray features in the near field of a flow-blurring injector investigated by high-speed visualization and time-resolved PIV". Experiments in Fluids, 56(5), may, p. 103.

[5] Khan, M. A., Gadgil, H., and Kumar, S., 2019. "Influence of liquid properties on atomization characteristics of flow-blurring injector at ultra-low flow rates". Energy, 171, mar, pp. 1-13.

[6] Murugan, R., and Kolhe, P. S., 2021. "Experimental investigation into flow blurring atomization". Experimental Thermal and Fluid Science, 120(May 2020), p. 110240.

[7] Español, P., and Revenga, M., 2003. "Smoothed dissipative particle dynamics". Phys. Rev. E, 67, Feb, p. 026705.

[8] Hu, X. Y., and Adams, N. A., 2006. "A multi-phase sph method for macroscopic and mesoscopic flows". Journal of Computational Physics, 213(2), pp. $844-861$.

[9] Monaghan, J., 1992. "Smoothed particle hydrodynamics". Annual Review of Astronomy and Astrophysics, 30(1), pp. 543-574.

[10] Liu, M. B., and Liu, G. R., 2010. "Smoothed particle hydrodynamics (sph): an overview and recent developments". Archives of Computational Methods in Engineering, 17(1), pp. 25-76.

[11] Adami, S., Hu, X. Y., and Adams, N. A., 2010. "A new surface-tension formulation for multiphase $\{\mathrm{SPH}\}$ using a reproducing divergence approximation". Journal of Computational Physics, 229(13), pp. $5011-5021$.

[12] Chaussonnet, G., Koch, R., Bauer, H.-J., Sänger, A., Jakobs, T., and Kolb, T., 2018. "Smoothed Particle Hydrodynamics Simulation of an Air-Assisted Atomizer Operating at High Pressure: Influence of Non-Newtonian Effects". Journal of Fluids Engineering, 140(6), jan.

[13] Dauch, T., Ates, C., Rapp, T., Keller, M., Chaussonnet, G., Kaden, J., Okraschevski, M., Koch, R., Dachsbacher, C., and Bauer, H.-J., 2019. "Analyzing the interaction of vortex and gas-liquid interface dynamics in fuel spray nozzles by means of Lagrangian-coherent structures (2D)". Energies, 12(13).

[14] Braun, S., Wieth, L., Holz, S., Dauch, T. F., Keller, M. C., Chaussonnet, G., Gepperth, S., Koch, R., and Bauer, H.-J., 2019. "Numerical prediction of air-assisted primary atomization using Smoothed Particle Hydrodynamics". International Journal of Multiphase Flow, 114, pp. 303-315.

[15] Chaussonnet, G., Dauch, T., Keller, M., Okraschevski, M., Ates, C., Schwitzke, C., Koch, R., and Bauer, H. J., 2020. "Progress in the Smoothed Particle Hydrodynamics Method to Simulate and Post-process Numerical Simulations of Annular Airblast Atomizers". Flow, Turbulence and Combustion, 105(4), nov, pp. 1119-1147.

[16] Haller, G., 2005. "An objective definition of a vortex". Journal of fluid mechanics, 525, pp. 1-26.

[17] Dauch, T. F., Braun, S., Wieth, L., Chaussonnet, G., Keller, M. C., Koch, R., and H.-J., B., 2017. "Computational prediction of primary breakup in fuel spray nozzles for aero-engine combustors". In ILASS Europe, 29th Annual Conference on Liquid Atomization and Spray Systems, Valencia, Spain. 